\title{
POLYNOMIAL DECAY OF CORRELATIONS FOR A CLASS OF SMOOTH FLOWS ON THE TWO TORUS
}

\author{
BY BASSAM FAYAD
}

\begin{abstract}
Kočergin introduced in 1975 a class of smooth flows on the two torus that are mixing. When these flows have one fixed point, they can be viewed as special flows over an irrational rotation of the circle, with a ceiling function having a powerlike singularity. Under a Diophantine condition on the rotation's angle, we prove that the special flows actually have a $t^{-\eta}$-speed of mixing, for some $\eta>0$.

RÉSUmÉ (Décroissance polynomiale des corrélations pour une classe de flots lisses sur $\mathbb{T}^{2}$ )

Kočergin a introduit en 1975 une classe de flots $C^{\infty}$ sur le tore à deux dimensions qui sont mélangeants. Quand ces flots ont un seul point fixe, ils correspondent à des flots spéciaux au-dessus d'une rotation irrationnelle du cercle, dont la fonction de suspension présente une singularité en puissance fractionnaire. Sous une condition diophantienne sur l'angle de la rotation, on prouve que ces flots spéciaux ont une vitesse de mélange en $t^{-\eta}$, pour un certain $\eta>0$.
\end{abstract}

\section{Introduction}

1.1. Kočergin gave in [5], examples of $C^{\infty}$ measure-preserving flows on the two torus that are mixing. He starts by proving that special flows over irrational rotations of the circle (or over interval exchange transformations) with

Texte reçu le 16 mai 2000, révisé le 2 novembre 2000, accepté le 5 janvier 2001

Bassam FAYAD, Centre de Mathématiques, UMR 7640 du CNRS, École polytechnique, 91128 Palaiseau Cedex (France) • E-mail : fayadb@math.polytechnique.fr

2000 Mathematics Subject Classification. - 37E35.

Key words and phrases. - Flows on the torus, special flows, speed of mixing, correlations. Part of this work was carried on during my stay at CAMS (Beirut, Lebanon) in the summer of 1999. I wish to thank the members of this institution for their warm hospitality. 
a ceiling function having a power-like singularity are mixing. It is possible to identify these special flows with smooth flows on the two torus, having one fixed point or more. In the same article, Kočergin describes in some examples how to realize this identification by smoothly gluing to an irrational flow $R_{t(1, \alpha)}$ a small neighborhood of the fixed point of an adequatly chosen Hamiltonian flow in the plane. We will prove that under a Diophantine condition on the rotation number, the special flow has a polynomial decay of correlations between rectangles. We will see later why the arithmetical condition is required.

1.2. First we give the definition of a special flow over an irrational rotation. Given a strictly positive function $\varphi \in L^{1}\left(\mathbb{T}^{1}\right)$, the special flow constructed over $R_{\alpha}$ and under the function $\varphi$ is the quotient flow of the action

$$
\begin{aligned}
\mathbb{T}^{1} \times \mathbb{R} & \longrightarrow \mathbb{T}^{1} \times \mathbb{R}, \\
(x, s) & \longrightarrow(x, s+t),
\end{aligned}
$$

by the relation

$$
(x, s+\varphi(x)) \sim\left(R_{\alpha}(x), s\right) .
$$

This flow acts on the space

$$
M_{R_{\alpha}, \varphi}=\mathbb{T}^{1} \times \mathbb{R} / \sim,
$$

is uniquely ergodic and preserves the normalized Lebesgue measure on $M_{R_{\alpha}, \varphi}$, i.e. the product of the Haar measure on the basis $\mathbb{T}^{1}$ with the Lebesgue measure on the fibers divided by the constant $\int_{\mathbb{T}^{1}} \varphi(x) \mathrm{d} x$. In the sequel, we will simply denote by $M$ the space $M_{R_{\alpha}, \varphi}$, and by $\mu$ the invariant measure described above.

We call rectangles in $M$ the sets

$$
B=\bigcup_{t=t_{0}}^{t_{0}+\ell} T^{t}(I),
$$

when the union is disjoint and $I$ is an interval of $\mathbb{T}^{1}, t_{0} \in \mathbb{R}$ and $\ell \in \mathbb{R}_{+}^{*}$.

It is immediate that the collection of rectangles generates the $\sigma$-algebra of Borel sets on $M$. There is of course a slight abuse in calling rectangles the latter sets, because under the action of the flow, when $t_{0}$ or $\ell$ are large, they get distorted and do not have rectangular shapes anymore. Nevertheless, when $t_{0}=0$ and $\ell \leq \inf _{\mathbb{T}^{1}} \varphi$, we have real rectangles that we call rectangles on the basis.

1.3. Description of the flow under consideration. - In what follows we will consider the special flow $\left\{T^{t}\right\}$ constructed over an irrational rotation $R_{\alpha}$, and under a ceiling function $\varphi$.

We assume the following hypothesis on $\varphi$ :

TOME $129-2001-\mathrm{N}^{\mathrm{O}} 4$ 
- $\varphi \in C^{3}(\mathbb{T} /\{0\})$, and $\inf _{\mathbb{T}^{1}} \varphi=c>0$;

- there exists $0<\gamma<1$ such that

$$
\begin{array}{ll}
\lim _{x \rightarrow 0^{+}} \frac{\varphi(x)}{x^{-\gamma}}=1, & \lim _{x \rightarrow 0^{-}} \frac{\varphi(x)}{(-x)^{-\gamma}}=1, \\
\lim _{x \rightarrow 0^{+}} \frac{\varphi^{\prime}(x)}{-\gamma x^{-\gamma-1}}=1, & \lim _{x \rightarrow 0^{-}} \frac{\varphi^{\prime}(x)}{\gamma(-x)^{-\gamma-1}}=1, \\
\lim _{x \rightarrow 0^{+}} \frac{\varphi^{\prime \prime}(x)}{\gamma(\gamma+1) x^{-\gamma-2}}=1, & \lim _{x \rightarrow 0^{-}} \frac{\varphi^{\prime \prime}(x)}{\gamma(\gamma+1)(-x)^{-\gamma-2}}=1 .
\end{array}
$$

For commodity, we will also suppose that $\int_{\mathbb{T}^{1}} \varphi(x) \mathrm{d} x=1$.

It follows from Kočergin's result that these special flows, for any $\alpha$ and any $\gamma \in] 0,1[$, are mixing. But to force estimates on the decay of correlations, our techniques require that the exponent should be at least less than $\frac{1}{2}$, and we will assume $\gamma \leq \frac{2}{5}$.

REMARK. - The exponent obtained in one of the smooth examples given by Kočergin is $\gamma=\frac{1}{3}<\frac{2}{5}$.

As for the rotation number $\alpha$, we require that the sequence $\left\{q_{n}\right\}_{n \in \mathbb{N}}$ of denominators of its convergents satisfies for some positive constant $C_{\alpha}$

$$
q_{n+1} \leq C_{\alpha} q_{n}{ }^{1+\epsilon},
$$

where $\epsilon$ is small compared to $\gamma, \epsilon=\frac{1}{100} \gamma$ being enough for our purpose.

Finally, we introduce the number

$$
\eta:=\frac{1}{50} \gamma
$$

In the sequel, we will often use the fact that $2 \epsilon \leq \eta \ll \gamma$.

1.4. Statement. - Under the above assumptions on $\alpha$ and $\varphi$, we will show the following:

Theorem 1.1. - For any two rectangles $A$ and $B$, for any $\eta_{0}<\eta$, we have for $t$ large enough

$$
\left|\mu\left(A \cap T^{-t} B\right)-\mu(A) \mu(B)\right| \leq \frac{1}{t^{\eta_{0}}} .
$$

In the statement, the rectangles and the measure $\mu$ are as defined in (1.2). BULletin DE LA SOCiÉtÉ MATHÉmATiQUe DE FRANCE 
1.5. Remarks. - For a fixed $\epsilon>0$, the set of rotation numbers satisfying a Diophantine condition $(\mathrm{CD}-\epsilon)$ is of total Lebesgue measure.

To simplify the presentation we considered only one power-like and symmetrical singularity

$$
\varphi(x) \underset{0}{\sim} \varphi(-x) \underset{0}{\sim}|x|^{-\gamma} .
$$

From the proof it will appear clearly that the same result holds when there are finitely many singularities, and not all of them being necessarily power-like (some of them could be logarithmic for example), under the condition that the strongest one should be power-like (with exponent $\gamma \leq \frac{2}{5}$ ). Furthermore, our assumption of symmetry is not necessary, to the contrary, symmetry plays in general against mixing. When the singularity is logarithmic for example, the symmetry impedes mixing as proved by Kočergin in [4]; while Khanin and Sinai proved mixing in the case of asymmetrical logarithmic singularities [3].

Our estimates are far from optimal and $\eta=\frac{\gamma}{50}$ is certainly not the best polynomial rate of decay one can obtain. A faster speed of mixing than $t^{-\frac{1}{2}-\epsilon}$ would be very interesting because it would imply a Lebesgue spectrum for the flow. But since it appeared very hard to obtain faster decay than $t^{-\frac{1}{2}}$ by the techniques involved in this paper we wrote the proof with the above $\eta=\frac{\gamma}{50}$.

Correlations between functions. - Through the proof of the theorem, it will appear that (1) is valid when $t \in\left[q_{n}, q_{n+1}\right], n$ sufficiently large, for any pair of squares $A$ and $B$ with side of length equal to $q_{n}^{-\eta}$. Hence we could establish for any couple of complex functions of class $C^{1}$ on $\mathbb{T}^{2}$, the same decay of correlations obtained for rectangles.

1.6. Plan of the proof. — The property underlying mixing for a special flow over a rigid transformation is the uniform stretch of the Birkhoff sums of the ceiling function,

$$
\varphi_{m}(x):=\varphi(x)+\varphi\left(R_{\alpha}(x)\right)+\cdots+\varphi\left(R_{\alpha}^{m-1}(x)\right) .
$$

When the Birkhoff sums have large derivatives, the image of a small interval $J \in$ $\mathbb{T}^{1}$ by the flow is stretched with time in the vertical direction along the fibers, and as $t$ tends to infinity the interval actually breaks down into a lot of almost vertical curves whose projections on the circle follow the trajectory of $R_{\alpha}$. By unique ergodicity of the rotation these projections become more uniformly distributed on the circle as their number increases, and so will be $T^{t}(J)$ in the whole space (see [6], [5], [3], [2] and [1]).

For each $t$, we want to cover the circle excepted a small set with intervals being stretched as described above (here, we want the exceptional set to have measure less than $t^{-\eta}$ ). The first intervals to be over ruled are those that come too close to the singularity before time $t$ and eventually get trapped in its neighborhood (Lemma 2.3). Other intervals must be automatically discarded,

TOME $129-2001-\mathrm{N}^{\mathrm{O}} 4$ 
those where there is no stretch at all, i.e. where the first derivative of $\varphi_{m}$ is small (the singularity being symmetric this likely occurs): a lower bound on the second derivatives due to the convex average behavior of $\varphi$ will allow us to estimate the size of such bad intervals (Lemma 2.5 and Step 2 in Section 3.1).

For the remaining part of the circle, we seek a good control on the stretch of $\varphi_{m}$, when $m$ is comparable with $t$, and here the Diophantine condition on $\alpha$ is required to insure the uniformity of stretch (Lemmas 2.4 and 2.5). Still, uniform stretch (Properties (P1)- $\left(\mathrm{P} 2^{\prime}\right)$ in (3.1)) of an interval $J$ is not enough by itself to estimate the asymptotic repartition of $T^{t}(J)$ in the space as $t$ goes to infinity. We need in addition to make sure that the pieces of $T^{t}(J)$ (those almost vertical curves) do not enter in a too small neighborhood of the singularity, otherwise a lot of measure can be lost there (See (2.4)).

A "good" partition is finally constructed for each time $t$ (Proposition 3.1) and Lemmas 3.3 and 3.2 give a precise description of $T^{t}(J)$, for an interval $J$ in this partition.

To conclude, we need a good estimate on the asymptotic distribution of the trajectories of the rotation on the circle that we obtain using again the Diophantine condition on $\alpha$.

\section{Preliminary estimates and lemmas}

2.1. A Fubini Lemma. - We begin by a Fubini lemma that reduces our problem to studying the image under the special flow of intervals on its basis $\mathbb{T}^{1}$.

Given $\nu>0$ and a finite partial partition $\mathcal{P}=\left\{I_{0}, \ldots, I_{m}\right\}$ of $\mathbb{T}^{1}$, we say that $\mathcal{P}$ is $\nu$-fine if, for any interval $I$ on the circle, there exists a collection of atoms from $\mathcal{P}$ such that the symmetrical difference between their union and $I$ has Lebesgue measure less than $\nu$.

We recall that a rectangle on the basis is a subset $B=\bigcup_{0 \leq t \leq \ell} T^{t}(I)$, where $I$ is an interval of $\mathbb{T}^{1}$ and $\ell \in \mathbb{R}_{+}^{*}$ is the height of $B$. We also recall that $\eta$ is the fixed number $\frac{1}{50} \gamma\left(2 \epsilon \leq \eta \ll \gamma \leq \frac{2}{5}\right)$.

LEMmA 2.1. - If there exist partial partitions of $\mathbb{T}^{1}, \mathcal{P}_{t}$, that are $t^{-\eta}$-fine and such that for any rectangle $B$ on the basis with height less than $c=\inf _{\mathbb{T}^{1}} \varphi$, we have when $t$ is large enough

$$
\left|\lambda\left(J_{i}^{(t)} \cap T^{-t} B\right)-\lambda\left(J_{i}^{(t)}\right) \mu(B)\right| \leq t^{-\eta} \lambda\left(J_{i}^{(t)}\right),
$$

for every $J_{i}^{(t)} \in \mathcal{P}_{t}$, then Theorem 1.1 is true.

In the statement of the Lemma, $\lambda$ is the Haar measure on the circle and $\mu$ is the normalized measure on $M$ invariant by the special flow.

BULLETIN DE LA SOCIÉtÉ MATHÉMATIQUE DE FRANCE 
Proof. - Take two rectangles in $M$,

$$
A=\bigcup_{t_{1} \leq s \leq t_{1}+\ell_{1}} T^{s}(I) \text { and } B=\bigcup_{t_{2} \leq s \leq t_{2}+\ell_{2}} T^{s}\left(I^{\prime}\right)
$$

$\left(\ell_{1}\right.$ and $\ell_{2}$ are the heights of $A$ and $B$ ). Since the rectangle $B$ can always be decomposed into a finite disjoint union of rectangles with height less than $c$, it is enough to prove Theorem 1.1 when $\ell_{2}=\delta<c$. On the other hand one has

$$
\mu\left(A \cap T^{-t} B\right)=\mu\left(A \cap T^{-\left(t-t_{2}\right)} T^{-t_{2}} B\right),
$$

hence we can also assume for $B$ that $t_{2}=0$. From the hypothesis of the Lemma, when $t$ is large enough, there exist atoms $J_{1}^{(t)}, \ldots, J_{l}^{(t)}$ from $\mathcal{P}_{t}$ such that

$$
\lambda\left(I \triangle \bigcup_{i=0}^{\ell} J_{i}^{(t)}\right) \leq t^{-\eta}
$$

and for each one of which (2) holds. Hence,

$$
\left|\lambda\left(I \cap T^{-t} B\right)-\lambda(I) \mu(B)\right| \leq 3 t^{-\eta} .
$$

Since $A=\bigcup_{t_{1}<s<t_{1}+\ell_{1}} T^{s}(I)$, we apply the Fubini lemma and obtain the estimation of the Theorem 1 when $t$ is large enough (we took $\eta_{0}<\eta$ to avoid having constants in the statement of the theorem). Of course the same conclusion holds if we establish (2) with some constant in front of $t^{-\eta}$.

2.2. Avoiding the singularity at time $t$. - We recall that

$$
\varphi_{m}(x)=\sum_{k=0}^{m-1} \varphi\left(R_{\alpha}^{k}(x)\right) .
$$

For any $x \in \mathbb{T}^{1}$ (a point on the basis), and any positive time $t$, there is a unique integer $m$ such that

$$
0 \leq t-\varphi_{m}(x)<\varphi\left(R_{\alpha}^{m}(x)\right) .
$$

We denote this integer by $N(x, t)$. Since $\inf _{\mathbb{T}^{1}} \varphi=c>0$, we have:

Lemma 2.2. - For every $x \in \mathbb{T}^{1}$ and any time $t$, we have

$$
N(x, t) \leq \frac{t}{c}
$$

Proof. - One has $t \geq \varphi_{N(x, t)}(x) \geq N(x, t) c$ by definition of $N(x, t)$.

Let $t \in\left[q_{n}, q_{n+1}\right]$. From the lemma above, we know we will avoid going too close to the singularity at time $t$ if we consider only the set

$$
F_{n}=\mathbb{T}^{1}-\bigcup_{k=0}^{2\left[q_{n+1} / c\right]} R_{\alpha}^{-k}\left[-\frac{1}{q_{n+1}^{1+\eta}}, \frac{1}{q_{n+1}^{1+\eta}}\right] .
$$

(The notation $[r]$ stands for the integer part of $r \in \mathbb{R}$.) For this set we can state the following, when $n$ is large enough

TOME $129-2001-\mathrm{N}^{\mathrm{O}} 4$ 
Lemma 2.3. - For any $x, y \in F_{n}$, for any $t \in\left[q_{n}, q_{n+1}\right]$, we have

$$
\begin{aligned}
|N(x, t)-N(y, t)| & \leq q_{n+1}^{\gamma+2 \eta}, \\
\frac{t}{2} \leq N(x, t) & \leq \frac{t}{c} .
\end{aligned}
$$

Proof. - By definition of the number $N(x, t)$, we have

$$
\left|t-\varphi_{N(x, t)}(x)\right| \leq \varphi\left(R_{\alpha}^{N(x, t)}(x)\right) .
$$

Since $x \in F_{n},(3)$ implies that $R_{\alpha}^{N(x, t)}(x) \in \mathbb{T}^{1}-\left[-q_{n+1}^{-1-\eta}, q_{n+1}^{-1-\eta}\right]$, hence

$$
\left|t-\varphi_{N(x, t)}(x)\right| \leq 2 q_{n+1}^{(1+\eta) \gamma}
$$

because $\varphi(|x|) \sim_{0}|x|^{-\gamma}$ and $\varphi \in C^{3}\left(\mathbb{T}^{1} / 0\right)$.

Now, on one hand $t \geq q_{n} \geq q_{n+1}{ }^{1 /(1+\epsilon)}$, and the other $\gamma \leq \frac{2}{5}, \epsilon \ll 1$ and $\eta \ll 1$, hence (6) leads to

$$
\varphi_{N(x, t)}(x) \geq \frac{9}{10} t .
$$

We want now a bound on $\left|\varphi_{m}(x)-m\right|$, when $x \in F_{n}$ and $m \leq 2 q_{n+1} / c$. First, we write $m^{\prime}$ s expansion:

$$
m=\sum_{s=0}^{\ell_{m}} m_{s} q_{s}, \quad 0 \leq m_{s} \leq a_{s+1},
$$

where $a_{s+1}$ denote the partial quotients of the continued fraction expansion of $\alpha$. Since $\alpha$ is $\epsilon$-Diophantine, $a_{s+1} \leq q_{s}^{\epsilon}$, by definition. Furthermore, because $m \leq 2 q_{n+1} / c$, we have $\ell_{m} \leq n+1$ and $\sup m_{s} \leq q_{n}^{\epsilon}$ in the expression of $m$. Let $\tilde{\varphi}$ be the function equal to $\varphi$ outside $\left[-q_{n+1}^{-1-\eta}, q_{n+1}^{-1-\eta}\right]$, constant on the latter interval and of integral one on the torus. Applying the Denjoy-Koksma inequality to $\tilde{\varphi}$ we have, for any $s \in \mathbb{N}$,

$$
\left|\tilde{\varphi}_{q_{s}}(x)-q_{s}\right| \leq \operatorname{Var} \tilde{\varphi},
$$

but the variations of $\tilde{\varphi}$ on the circle are of the order of $q_{n+1}^{(1+\eta) \gamma}$, hence

$$
\left|\tilde{\varphi}_{q_{s}}(x)-q_{s}\right| \leq C q_{n+1}^{(1+\eta) \gamma}
$$

where $C$ is a constant depending only on $\varphi$. Using $m$ 's expansion, we have for any $x$ on the circle

$$
\left|\tilde{\varphi}_{m}(x)-m\right| \leq C(n+2) q_{n}^{\epsilon} q_{n+1}^{(1+\eta) \gamma},
$$

so when $n$ is large enough, we have

$$
\left|\tilde{\varphi}_{m}(x)-m\right| \leq q_{n+1}^{(1+\eta) \gamma+2 \epsilon}
$$

when $n$ is large enough.

BULLETIN DE LA SOCIÉTÉ MATHÉMATIQUE DE FRANCE 
On the other hand, for $m \leq 2 q_{n+1} / c$ we have $R_{\alpha}^{m}(x) \in \mathbb{T}^{1}-\left[-q_{n+1}^{-1-\eta}, q_{n+1}^{-1-\eta}\right]$ for any $x \in F_{n}$, so $\tilde{\varphi}_{m}(x)=\varphi_{m}(x)$. The above equation then becomes for any $x \in F_{n}$ and $m \leq 2 q_{n+1} / c$

$$
\left|\varphi_{m}(x)-m\right| \leq q_{n+1}^{(1+\eta) \gamma+2 \epsilon}=o\left(q_{n+1}^{\gamma+2 \eta}\right) .
$$

Finally, (4) follows from (6) and (10), while (5) follows from (7) and (10).

2.3. Stretch estimates. - To obtain sharp estimates for the stretch requires a good control on the first and second derivatives of the Birkhoff sums of $\varphi$. From Lemma 2.3 we know that when $t \in\left[q_{n}, q_{n+1}\right]$, we have to consider those sums for $m \in\left[q_{n} / 2, q_{n+1} / c\right]$. A lower bound on the first derivatives will guarantee good stretch and an upper bound on the second will insure its uniformity. Here again, we will exploit the Diophantine condition on $\alpha$. Nevertheless, because the singularity is symmetrical, we will not be able to minorate directly the first derivatives of $\varphi$. It is a fact in this case that at some points of the circle the Birkhoff sums are not stretching at all. The lower bound on the second derivatives that comes from the convex average behavior of $\varphi$ will allow us to overcome this difficulty.

We will denote the first and second derivatives of $\varphi_{m}$ by $\varphi_{m}^{\prime}$ and $\varphi_{m}^{\prime \prime}$. When $n$ is sufficiently large, we have

Lemma 2.4. - For any $m \leq 2 q_{n+1} / c$ and $x \in F_{n}$,

$$
\left|\varphi_{m}^{\prime}(x)\right| \leq q_{n+1}^{1+\gamma+2 \eta} .
$$

We also have, for sufficiently large $n$,

LEMMA 2.5. - For any $m \in\left[q_{n} / 2,2 q_{n+1} / c\right]$ and $x \in F_{n}$,

$$
q_{n+1}^{2+\gamma-3 \eta} \leq \varphi_{m}^{\prime \prime}(x) \leq q_{n+1}^{2+\gamma+3 \eta}
$$

Proof of Lemma 2.4. - Write $\varphi=\underline{\varphi}+\bar{\varphi}$, where

$$
\bar{\varphi}=\chi_{\left[-1 / q_{n+1}, 1 / q_{n+1}\right]} \varphi
$$

$\left(\chi_{L}\right.$ being the characteristic function of a set $L$ ). Reasoning exactly as in the proof of (9), the Diophantine condition on $\alpha$ and the Denjoy-Koksma inequality imply for $m \leq 2 q_{n+1} / c$ and for any $x \in \mathbb{T}^{1}$

$$
\left|\underline{\varphi}_{m}^{\prime}(x)-m \int_{\mathbb{T}^{1}} \underline{\varphi}^{\prime}(y) \mathrm{d} y\right| \leq q_{n+1}^{1+\gamma+2 \epsilon} .
$$

But $\int_{\mathbb{T}^{1}} \underline{\varphi}^{\prime}(y) \mathrm{d} y=O\left(q_{n+1}^{\gamma}\right)$, hence $m \int_{\mathbb{T}^{1}} \underline{\varphi}^{\prime}(y) \mathrm{d} y=O\left(q_{n+1}^{1+\gamma}\right)$ and we have for sufficiently large $n$

$$
\left|\underline{\varphi}_{m}^{\prime}(x)\right| \leq 2 q_{n+1}^{1+\gamma+2 \epsilon} .
$$

On the other hand under $m$ iterations, $m \leq 2 q_{n+1} / c$, any point $x$ enters less than $8[1 / c]$ times in the interval $I_{n}:=\left[-1 / q_{n+1}, 1 / q_{n+1}\right]$ (where $[r]$ denotes

TOME $129-2001-\mathrm{N}^{\mathrm{O}} 4$ 
the integer part of $r \in \mathbb{R}$. We used the fact that successive $\left[\frac{1}{4} q_{n+1}\right]$ iterates of $I_{n}$ by $R_{\alpha}$ are disjoint). Meanwhile, $x \in F_{n}$ and $R_{\alpha}^{m}(x)$ still remains outside $\left[-q_{n+1}^{-1-\eta}, q_{n+1}^{-1-\eta}\right]$ for $m \leq 2 q_{n+1} / c$, hence

$$
\left|\bar{\varphi}_{m}^{\prime}(x)\right| \leq \frac{9}{c} \gamma q_{n+1}^{(1+\eta)(1+\gamma)}
$$

because $\left|\varphi^{\prime}(x)\right| \sim \gamma|x|^{-\gamma-1}$ in the neighborhood of 0 .

Lemma 2.4 then follows from (13) and (14) when $n$ is sufficiently large since $2 \eta>\eta(1+\gamma)>2 \epsilon$.

Proof of Lemma 2.5. - The right hand side in (12) is obtained in exactly the same way as (11). For the left hand side consider the decomposition $\varphi=$ $\underline{\varphi}+\bar{\varphi}$, with this time $\bar{\varphi}=\chi_{K} \varphi$, where $K$ is a fixed interval containing the singularity and on which the second derivative is always positive. It is possible to do so since we assumed $\varphi^{\prime \prime}(|x|) \sim_{0} \gamma(\gamma+1)|x|^{-2-\gamma}$. Clearly, for any $m \in \mathbb{N}$ and $x \in \mathbb{T}^{1}$,

$$
\left|\underline{\varphi}_{m}^{\prime \prime}(x)\right| \leq C m,
$$

where $C$ here is the supremum of the second derivative of $\varphi$ outside $K$.

On the other hand in $m$ iterations, $m \geq \frac{1}{2} q_{n} \geq q_{n-1}$, the orbit of any point $x$ enters at least once in the interval $\left[-1 / q_{n-1}, 1 / q_{n-1}\right]$, since the union of the first $q_{n-1}$ inverse iterates of this interval cover the circle. From the hypothesis on the singularity of $\varphi$ and the Diophantine condition on $\alpha$ we conclude that for $x \in \mathbb{T}^{1}, m \in\left[\frac{1}{2} q_{n}, q_{n+1}\right]$

$$
\bar{\varphi}_{m}^{\prime \prime}(x) \geq q_{n-1}^{2+\gamma} \geq q_{n+1}^{(2+\gamma) /(1+\epsilon)^{2}} .
$$

Since $(1+\epsilon)^{-2}$ is of the order of $1-\eta$, and $(2+\gamma)(1-\eta)>2+\gamma-3 \eta$, the last equation and (15) yield the left hand side in (12).

2.4. Central lemma for the asymptotic distribution of the pieces of $\boldsymbol{T}^{\boldsymbol{t}}(\boldsymbol{J})$. - In the introduction we explained how an interval $I$ breaks down under stretch into many almost vertical curves whose projection on the basis follows the trajectory of the rotation.

From Lemma 2.3, we know more or less where do these parts of $T^{t}(J)$ end up at time $t$. To have sharp estimates on their distribution in the whole space, we want to make sure that the parts stay relatively far from the singularity. We will define to this end, for each time $t \in\left[q_{n}, q_{n+1}\right]$, a subset of $F_{n}$ (see (18)) and a partition of this subset into intervals (see (3.1)) that after they get stretched will have a good asymptotic distribution in the space.

The following lemma, is essential in establishing the description of $T^{t}(J)$ that will given in Lemma 3.2 and Lemma 3.3.

BULletin DE LA SOCIÉtÉ MATHÉMATIQUE DE FRANCE 
Let $\beta$ be a positive number such that

$$
\begin{gathered}
\beta>\gamma+4 \eta, \\
\beta \gamma<\gamma-18 \eta,
\end{gathered}
$$

We can resume the conditions (16) and (17) by asking that

$$
\gamma+4 \eta<\beta<1-18 \frac{\eta}{\gamma}
$$

which is easy to realize for some $\beta$ since $\eta \ll \gamma$, and $\gamma \leq \frac{2}{5}$ is also far from 1 .

2.4.1. Denote by $\gamma^{\prime}$ the exponent $\gamma+2 \eta$ that appeared in (4).

2.4.2. Define the set

$$
T_{n, \beta}=\mathbb{T}^{1}-\bigcup_{k=-2\left[q_{n+1}^{\gamma^{\prime}}\right]}^{2\left[q_{n+1}^{\gamma^{\prime}}\right]} R_{\alpha}^{-k}\left[-\frac{1}{q_{n+1} \beta}, \frac{1}{q_{n+1} \beta}\right] .
$$

REMARK 2.6. - With our choice of $\beta$, we clearly have $\lambda\left(T_{n, \beta}\right) \geq 1-8 q_{n+1}^{-2 \eta}$.

Lemma 2.7. - For any $x \in T_{n, \beta}$ and for $D \in \mathbb{N}$ such that

$$
q_{n+1}^{\gamma-15 \eta} \leq D \leq 2 q_{n+1}^{\gamma^{\prime}},
$$

we have for sufficiently large $n$

$$
\begin{gathered}
\left|\varphi_{D}(x)-D\right| \leq q_{n+1}^{-\eta} D \\
\left|\varphi_{D}^{\prime}(x)\right| \leq q_{n+1}^{(\gamma+1) \beta+\eta} .
\end{gathered}
$$

Proof. - Reasoning the same way as in the previous lemmas we obtain, for $D \leq q_{n+1}^{\gamma^{\prime}}$ and $x \in T_{n, \beta}$, equation (20) and

$$
\left|\varphi_{D}(x)-D\right| \leq q_{n+1}^{\gamma \beta+2 \epsilon}
$$

With our choice of $\beta$ (cf. (17)), we have $\gamma \beta+2 \epsilon \leq \gamma \beta+\eta<\gamma-16 \eta$. Hence, the lower bound on $D$ and (21) imply (19).

\section{Proof of the theorem on correlations.}

3.1. Construction of the good partition at time $\boldsymbol{t}$. - We fix now $t$ in $\left[q_{n}, q_{n+1}\right]$ and pick an arbitrary $x_{0} \in F_{n}$. Define

$$
F_{t, \beta}=F_{n}-\bigcup_{k=N\left(x_{0}, t\right)-2\left[q_{n+1}^{\gamma^{\prime}}\right]}^{N\left(x_{0}, t\right)+2\left[q_{n+1}^{\gamma^{\prime}}\right]} R_{\alpha}^{-k}\left[-\frac{1}{q_{n+1} \beta}, \frac{1}{q_{n+1} \beta}\right] .
$$

Proposition 3.1. - It is possible to construct a partial partition $\mathcal{P}_{t}$ of $F_{t, \beta}$ consisting of intervals, with the following properties:

TOME $129-2001-\mathrm{N}^{\mathrm{O}} 4$ 
(P0) the partial partition $\mathcal{P}_{t}$ is $t^{-\eta}$-fine on the circle;

(P1) the sizes of the intervals in $\mathcal{P}_{t}$ vary between $q_{n+1}^{-1-9 \eta}$ and $2 q_{n+1}^{-1-9 \eta}$;

(P2) for any $J \in \mathcal{P}_{t}$ and any $m \in\left[N\left(x_{0}, t\right)-q_{n+1}^{\gamma^{\prime}}, N\left(x_{0}, t\right)+q_{n+1}^{\gamma^{\prime}}\right]$

$$
\inf _{x \in J}\left|\varphi_{m}^{\prime}(x)\right| \geq q_{n+1}^{1+\gamma-5 \eta}
$$

Claim. - As a direct consequence of (P1), (P2) and the upper bound in (12) we will have for large $n$ :

$\left(\mathrm{P} 2^{\prime}\right)$ For any $J \in \mathcal{P}_{t}$ and any $m \in\left[N\left(x_{0}, t\right)-q_{n+1}^{\gamma^{\prime}}, N\left(x_{0}, t\right)+q_{n+1}^{\gamma^{\prime}}\right]$,

$$
\sup _{x \in J}\left|\varphi_{m}^{\prime \prime}(x)\right| \cdot|J| \leq 2 q_{n+1}^{-\eta} \inf _{x \in J}\left|\varphi_{m}^{\prime}(x)\right|
$$

Proof of the claim. - Since $N\left(x_{0}, t\right) \leq q_{n+1} / c, m$ satisfies the hypothesis of Lemma 2.5 and we have $\sup _{x \in J}\left|\varphi_{m}^{\prime \prime}(x) \| J\right| \leq q_{n+1}^{2+\gamma+3 \eta} 2 q_{n+1}^{-1-9 \eta}$.

Proof of Proposition 3.1. - We will do the construction in three steps.

First step. - We cover the circle with disjoint intervals of sizes varying between $q_{n+1}^{-1-\eta}$ and $2 q_{n+1}^{-1-\eta}$. (We do not care about the ending points of the intervals because their total measure is always zero and we will indifferently talk about segments or intervals.) In this first step we just take away from the above intervals these that are not completely included in $F_{t, \beta}$, or equivalently, these that intersect either

$$
\bigcup_{k=0}^{2\left[q_{n+1} / c\right]} R_{\alpha}^{-k}\left[-1 / q_{n+1}^{1+\eta}, 1 / q_{n+1}^{1+\eta}\right]
$$

or

$$
\bigcup_{k=N\left(x_{0}, t\right)-2\left[q_{n+1}^{\gamma^{\prime}}\right]}^{N\left(x_{0}, t\right)+2\left[q_{n+1}^{\gamma^{\prime}}\right]} R_{\alpha}^{-k}\left[-1 / q_{n+1}{ }^{\beta}, 1 / q_{n+1}{ }^{\beta}\right] .
$$

The total measure of the discarded segments is bounded by

$$
2\left[\frac{q_{n+1}}{c}\right] \frac{2}{q_{n+1}^{1+\eta}}+4\left[\frac{q_{n+1}}{c}\right] \frac{2}{q_{n+1}^{1+\eta}}+4\left[q_{n+1}^{\gamma^{\prime}}\right] \frac{2}{q_{n+1} \beta}+8\left[q_{n+1}^{\gamma^{\prime}}\right] \frac{2}{q_{n+1}^{1+\eta}}=O\left(q_{n+1}^{-\eta}\right)
$$

due to our choice of $\beta$. The partial partition we obtained is hence $q_{n+1}^{-\eta}$-fine (up to a constant).

Second step. - Consider any interval $L$ of the ones we kept in the first step. It follows from Lemma 2.2 and Lemma 2.5 that for $m_{0}=N\left(x_{0}, t\right), \varphi_{m_{0}}^{\prime \prime}$ is greater than $q_{n+1}^{2+\gamma-3 \eta}$ on $L$. So, after taking off eventually an interval of size $4 q_{n+1}^{-1-2 \eta}$ there will remain from $L$ one or two intervals, $L_{\ell}$ and $L_{r}$, where $\left|\varphi_{m_{0}}^{\prime}\right|$ is always greater than $2 q_{n+1}^{1+\gamma-5 \eta}$. If one of those intervals, $L_{\ell}$ or $L_{r}$ has size less than $q_{n+1}^{-1-2 \eta}$ we discard it (see Fig. 1 ).

BULLETIN DE LA SOCIÉtÉ MATHÉMATIQUE DE FRANCE 


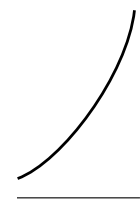

$L$
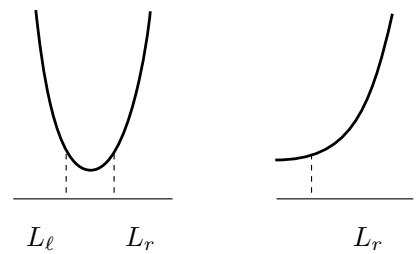

FiguRE 1. Different configurations for $\varphi_{m}$ over $L$

The total measure we might loose by this operation on $L$ is $5 q_{n+1}^{-1-2 \eta}$ which is at most a proportion $5 q_{n+1}^{-\eta}$ of the interval $L$. Hence we remain with a partial partition that is $q_{n+1}^{-\eta}$-fine (up to a constant).

Moreover, we claim that the bound on $\varphi_{m_{0}}^{\prime}$ leads to (P2). Indeed, for any $m \in\left[m_{0}-q_{n+1}^{\gamma^{\prime}}, m_{0}+q_{n+1}^{\gamma^{\prime}}\right]$,

$$
\varphi_{m}^{\prime}(x)=\varphi_{m-m_{0}}^{\prime}\left(R_{\alpha}^{m_{0}} x\right)+\varphi_{m_{0}}^{\prime}(x) ;
$$

but $L_{r}$ and $L_{\ell}$ are in $F_{t, \beta}$, so $R_{\alpha}^{m_{0}}(x) \in T_{n, \beta}$, therefore (20), of Lemma 2.7, implies that

$$
\varphi_{m-m_{0}}^{\prime}\left(R_{\alpha}^{m_{0}} x\right) \leq q_{n+1}^{(\gamma+1) \beta+\eta}
$$

when $x \in L_{r}, x \in L_{\ell}$. From the choice of $\beta$ (cf. (17) it follows that $(\gamma+1) \beta+\eta<$ $1+\gamma-5 \eta$. Hence, $\varphi_{m-m_{0}}^{\prime}\left(R_{\alpha}^{m_{0}} x\right) \ll \varphi_{m_{0}}^{\prime}(x)$ and the claim follows.

Third step. - In this step we just divide each interval $L_{\ell}$ and $L_{r}$ we get from the first two steps into intervals of size varying between $q_{n+1}^{-1-9 \eta}$ and $2 q_{n+1}^{-1-9 \eta}$. It is possible to do so since the sizes of $L_{\ell}$ and $L_{r}$ are larger than $q_{n+1}^{-1-2 \eta}$. This gives $\mathcal{P}_{t}$.

3.2. Properties of a good interval. - From now on $J$ will designate an interval of $\mathcal{P}_{t}$. Fix a rectangle $B=\bigcup_{s=0}^{\delta} T^{s} V$. In the light of Lemma 2.1, we will finish if we prove that, under the conditions $(\mathrm{P} 1)-(\mathrm{P} 2)-\left(\mathrm{P} 2^{\prime}\right)$ on an interval $J$, the measure of the intersection $T^{t}(J) \cap B$ satisfies (2). Lemmas 3.2 and 3.4, that we will state below, will be the ingredients of our proof. Let $J \in \mathcal{P}_{t}$ be fixed

$$
J=\left[x_{1}, x_{2}\right], \quad m_{1}=N\left(x_{1}, t\right) m_{2}=N\left(x_{2}, t\right) .
$$

Since $x_{1}, x_{2} \in J \subset F_{n}$, Lemma 2.3 implies that

$$
m_{1}, m_{2} \in\left[N\left(x_{0}, t\right)-q_{n+1}^{\gamma^{\prime}}, N\left(x_{0}, t\right)+q_{n+1}^{\gamma^{\prime}}\right] .
$$

From (P2), it follows that $\varphi_{m}$ is monotone on $J$ and we will suppose $\varphi_{m_{1}}\left(x_{1}\right) \geq$ $\varphi_{m_{1}}\left(x_{2}\right)$, the other case being similar.

TOME $129-2001-\mathrm{N}^{\mathrm{O}} 4$ 
Define for $m \in \mathbb{N}$,

$$
\begin{aligned}
J_{m} & =\{x \in J ; N(x, t)=m\}, \\
& =\left\{x \in J ; 0 \leq t-\varphi_{m}(x)<\varphi\left(R_{\alpha}^{m}(x)\right)\right\} \\
J_{m, \delta} & =\left\{x \in J ; 0 \leq t-\varphi_{m}(x) \leq \delta\right\} .
\end{aligned}
$$

The set $J_{m}$ is the part of $J$ such that the projection of $T^{s}\left(J_{m}\right)$ on $\mathbb{T}^{1}$, when $s$ runs through $[0, t]$, is translated $m$ times by $R_{\alpha}$. And $J_{m, \delta}$ is the part of $J_{m}$ that lies at time $t$ in the band $\mathbb{T}^{1} \times[0, \delta]$.

Finally, denote by $\Delta \varphi_{m_{1 \mid J}}$ the quantity $\varphi_{m_{1}}\left(x_{1}\right)-\varphi_{m_{1}}\left(x_{2}\right)$.

For $m \in] m_{1}, m_{2}\left[\right.$, we have the following asymptotic estimate of $\lambda\left(J_{m, \delta}\right)(\lambda$ being the Haar measure on $\mathbb{T}^{1}$ )

LEMma 3.2. - For any $m$ such that $m_{1}<m<m_{2}$ it is true that

$$
\left|\lambda\left(J_{m, \delta}\right)-\frac{\delta|J|}{\Delta \varphi_{m_{1} \mid J}}\right| \leq q_{n+1}^{-\eta} \frac{\delta|J|}{\Delta \varphi_{m_{1} \mid J}} .
$$

For $m_{i}=m_{1}, m_{2}$ we have

$$
\lambda\left(J_{m_{i}, \delta}\right)-\frac{\delta|J|}{\Delta \varphi_{m_{1} \mid J}} \leq q_{n+1}^{-\eta} \frac{\delta|J|}{\Delta \varphi_{m_{1} \mid J}} .
$$

For $m$ not in $\left[m_{1}, m_{2}\right], J_{m, \delta}$ is empty.

Using Lemma 2.7 we will prove the following estimate on $m_{2}-m_{1}$ :

LEMMA 3.3. - We have

$$
\left|\frac{m_{2}-m_{1}}{\Delta \varphi_{m_{1} \mid J}}-1\right| \leq q_{n+1}^{-\eta} .
$$

Proof of Lemma 3.2. - First we will prove the following uniform estimate, for any $m \in\left[m_{1}, m_{2}\right]$ and for any $x \in J$

$$
\left|-\frac{1}{\varphi_{m}^{\prime}(x)}-\frac{|J|}{\Delta \varphi_{m_{1} \mid J}}\right| \leq 3 q_{n+1}^{-\eta} \frac{|J|}{\Delta \varphi_{m_{1} \mid J}} .
$$

By the mean value theorem, there exists $x_{1} \in J$ such that

$$
\Delta \varphi_{m_{1} \mid J}=\left|\varphi_{m_{1}}^{\prime}\left(x_{1}\right)\right| \cdot|J|,
$$

BULlETIN DE LA SOCIÉtÉ MATHÉMATIQUE DE FRANCE 
hence

$$
\begin{aligned}
E:=\left|-\frac{1}{\varphi_{m}^{\prime}(x)}-\frac{|J|}{\Delta \varphi_{m_{1} \mid J} \mid}\right| & =\frac{\left|\varphi_{m}^{\prime}(x)-\varphi_{m_{1}}^{\prime}\left(x_{1}\right)\right| \cdot|J|}{\left|\varphi_{m}^{\prime}(x)\right| \Delta \varphi_{m_{1} \mid J}} \\
& \leq \frac{\left|\varphi_{m}^{\prime}(x)-\varphi_{m}^{\prime}\left(x_{1}\right)\right| \cdot|J|}{\left|\varphi_{m}^{\prime}(x)\right| \Delta \varphi_{m_{1} \mid J}}+\frac{\left|\varphi_{m-m_{1}}^{\prime}\left(R_{\alpha}^{m_{1}} x_{1}\right)\right| \cdot|J|}{\left|\varphi_{m}^{\prime}(x)\right| \Delta \varphi_{m_{1} \mid J}} \\
& \leq \frac{\sup _{x \in J}\left|\varphi_{m}^{\prime \prime}(x)\right| \cdot|J|^{2}}{\left|\varphi_{m}^{\prime}(x)\right| \Delta \varphi_{m_{1} \mid J}}+\frac{\left|\varphi_{m-m_{1}}^{\prime}\left(R_{\alpha}^{m_{1}} x_{1}\right)\right| \cdot|J|}{\left|\varphi_{m}^{\prime}(x)\right| \Delta \varphi_{m_{1} \mid J}} .
\end{aligned}
$$

Using $\left(\mathrm{P} 2^{\prime}\right)$ we obtain

$$
E \leq 2 q_{n+1}^{-\eta} \frac{|J|}{\Delta \varphi_{m_{1} \mid J}}+\frac{\left|\varphi_{m-m_{1}}^{\prime}\left(R_{\alpha}^{m_{1}} x_{1}\right)\right| \cdot|J|}{\left|\varphi_{m}^{\prime}(x)\right| \Delta \varphi_{m_{1} \mid J}}
$$

Exactly as in our proof of (P2) in Proposition 3.1, it follows from (20) that

$$
\left|\varphi_{m-m_{1}}^{\prime}\left(R_{\alpha}^{m_{1}} x_{1}\right)\right| \leq q_{n+1}^{-\eta} \inf _{x \in J}\left|\varphi_{m}^{\prime}(x)\right| .
$$

This ends the proof of (26).

Now, for $m \in] m_{1}, m_{2}$ [ we have immediately from (26) that $\varphi_{m}$ is decreasing like $\varphi_{m_{1}}$. Also for such $m$, we have

$$
\begin{aligned}
& t-\varphi_{m}\left(x_{1}\right)<0, \\
& t-\varphi_{m}\left(x_{2}\right)>\delta,
\end{aligned}
$$

the first inequality following directly from $m_{1}:=N\left(x_{1}, t\right)$ (in the definition given in Section 2.2, $N(x, t)$ is the larger integer $m$ such that $\left.t-\varphi_{m}(x) \geq 0\right)$. For the second one, we use our assumption $\delta<c$ (indeed, $t-\varphi_{m_{2}}\left(x_{2}\right) \geq 0$ implies in this case (28) for any $\left.m \leq m_{2}-1\right)$.

In conclusion, for each $m \in] m_{1}, m_{2}$ [, by monotonicity of $\varphi_{m}$ and the mean value theorem, there exists $x_{m} \in J$ such that

$$
\lambda\left(J_{m, \delta}\right)=\frac{-\delta}{\varphi_{m}^{\prime}\left(x_{m}\right)} .
$$

Using (26) we obtain the first point of Lemma 3.2. The second point follows from (26) in a similar fashion. As for the third one we have to do the following argumentation: by definition of $m_{2}=N\left(x_{2}, t\right)$ one has $t-\varphi_{m_{2}+1}\left(x_{2}\right)<0$, but $\varphi_{m_{2}+1}$ is decreasing on $J$, therefore, for any $x \in J, t-\varphi_{m_{2}+1}(x)<0$. The same will clearly hold for any $m \geq m_{2}+1$, and $J_{m, \delta}$ will be empty for all $m>m_{2}$. When $m<m_{1}$, we use in addition that $\varphi$ is greater than $\delta$ to obtain $t-\varphi_{m}(x)>\delta$, for any $x \in J$, which implies $J_{m, \delta}$ is empty for $m<m_{1}$. The proof of Lemma 3.2 is accomplished.

Proof of Lemma 3.3. — Since $J \subset F_{t, \beta} \subset F_{n}$, Lemma 2.3 implies

$$
\left|m_{1}-N\left(x_{0}, t\right)\right| \leq q_{n+1}^{\gamma^{\prime}}
$$

TOME $129-2001-\mathrm{N}^{\mathrm{O}} 4$ 
hence, from Lemma 2.4 and conditions (P1) and (P2) we obtain

$$
q_{n+1}^{\gamma-14 \eta} \leq \Delta \varphi_{m_{1} \mid J} \leq 2 q_{n+1}^{\gamma-7 \eta} .
$$

Now $J \subset F_{t, \beta}$ implies

$$
R_{\alpha}^{m_{1}}(J) \subset T_{n, \beta} .
$$

In particular $\varphi\left(R_{\alpha}^{m_{1}}\left(x_{1}\right)\right) \leq 2 q_{n+1}^{\gamma \beta}$, and by the definition of $m_{1}$

$$
0 \leq t-\varphi_{m_{1}}\left(x_{1}\right) \leq \varphi\left(R_{\alpha}^{m_{1}}\left(x_{1}\right)\right) \leq 2 q_{n+1}^{\gamma \beta} .
$$

Together with the similar equation on $t-\varphi_{m_{2}}\left(x_{2}\right)$ this implies

$$
\left|\varphi_{m_{2}}\left(x_{2}\right)-\varphi_{m_{1}}\left(x_{1}\right)\right| \leq 4 q_{n+1}^{\gamma \beta} .
$$

On the other hand, we have

$$
\begin{aligned}
\varphi_{m_{2}}\left(x_{2}\right)-\varphi_{m_{1}}\left(x_{1}\right) & =\varphi_{m_{1}}\left(x_{2}\right)+\varphi_{m_{2}-m_{1}}\left(R_{\alpha}^{m_{1}} x_{2}\right)-\varphi_{m_{1}}\left(x_{1}\right), \\
& =\varphi_{m_{2}-m_{1}}\left(R_{\alpha}^{m_{1}} x_{2}\right)-\Delta \varphi_{m_{1} \mid J} .
\end{aligned}
$$

It follows from our choice of $\beta$ (17), that

$$
q_{n+1}^{\gamma \beta} \ll q_{n+1}^{-2 \eta} q_{n+1}^{\gamma-14 \eta} .
$$

Hence, the left hand side of (29) and (31) imply when $n$ is large

$$
\left|\varphi_{m_{2}-m_{1}}\left(R_{\alpha}^{m_{1}} x_{2}\right)-\Delta \varphi_{m_{1} \mid J}\right| \leq q_{n+1}^{-2 \eta} \Delta \varphi_{m_{1} \mid J} .
$$

From (30), we know that $R_{\alpha}^{m_{1}}\left(x_{2}\right) \in T_{n, \beta}$; to apply Lemma 2.7 and finish, we still need to prove that $q_{n+1}^{\gamma-15 \eta} \leq m_{2}-m_{1} \leq 2 q_{n+1}^{\gamma^{\prime}}$. From (29) and (32) we have

$$
\frac{1}{2} q_{n+1}^{\gamma-14 \eta} \leq \varphi_{m_{2}-m_{1}}\left(R_{\alpha}^{m_{1}} x_{2}\right) \leq 4 q_{n+1}^{\gamma-7 \eta}
$$

the right hand side of which implies that $m_{2}-m_{1} \leq c^{-1} 4 q_{n+1}^{\gamma-7 \eta} \ll q_{n+1}^{\gamma^{\prime}}$. Next, because $R_{\alpha}^{m_{1}}\left(x_{2}\right) \in T_{n, \beta},(21)$ is valid and implies the lower bound on $m_{2}-m_{1}$ from the lower bound on $\varphi_{m_{2}-m_{1}}\left(R_{\alpha}^{m_{1}} x_{2}\right)$ and $\Delta \varphi_{m_{1} \mid J}$.

\subsection{Asymptotic distribution on the circle of the rotation's orbit}

Since $\alpha$ is $\epsilon$-Diophantine, we have:

LEMMA 3.4. - Let $\chi_{V}$ be the characteristic function of an interval $V \in \mathbb{T}^{1}$, then for $N$ large enough, we have, for any $x \in \mathbb{T}^{1}$

$$
\left|\sum_{i=0}^{N-1} \chi_{V}\left(R_{\alpha}^{i}(x)\right)-N\right| V|| \leq N^{2 \epsilon}|V|,
$$

where $|V|=\lambda(V)$ is the Lebesgue measure of $V$.

BULLETIN DE LA SOCiÉTÉ MATHÉMATIQUe DE FRANCE 
Proof. - As we did in Section 2, we exploit the Diophantine condition on $\alpha$ to prove for any $x, x^{\prime} \in \mathbb{T}^{1}$ and $N$ large enough,

$$
\left|\sum_{i=0}^{N} \chi_{V}\left(R_{\alpha}^{i}(x)\right)-\sum_{i=0}^{N} \chi_{V}\left(R_{\alpha}^{i}\left(x^{\prime}\right)\right)\right| \leq N^{2 \epsilon}|V|,
$$

then we conclude using that $\int_{\mathbb{T}^{1}} \sum_{i=0}^{N} \chi_{V}\left(R_{\alpha}^{i}(x)\right) \mathrm{d} x=N|V|$.

3.4. End of the proof of the theorem. - Let $B=\bigcup_{s=0}^{\delta} T^{s}(V)$. We will prove, for $t$ large enough, the claim (2) for any given interval $J$ in the partition $\mathcal{P}_{t}$ constructed in Proposition 3.1. Define $V^{-} \subset V \subset V^{+}$to be the intervals obtained from $V$ by fixing its center and respectively shrinking and expanding the endpoints by a ratio $1+q_{n+1}^{-\eta}$. We will keep in mind that

$$
\begin{aligned}
& \left|V^{+}\right| \leq\left(1+q_{n+1}^{-\eta}\right)|V|, \\
& \left|V^{-}\right| \geq\left(1-2 q_{n+1}^{-\eta}\right)|V| .
\end{aligned}
$$

By definition (see (23)), $J_{m}$ is the part of $J$ such that $T^{t}\left(J_{m}\right)$ lies on the fibers over $R_{\alpha}^{m}(J)$. But the size of $J$ is less than $2 q_{n+1}^{-1-9 \eta}$ hence if $x_{0}$ is any fixed point in $J$ we have:

- If $R_{\alpha}^{m}\left(x_{0}\right) \in V^{-}$then $R_{\alpha}^{m}(J) \subset V$.

- If $R_{\alpha}^{m}(J) \bigcap V$ is non empty then $R_{\alpha}^{m}\left(x_{0}\right) \in V^{+}$.

On the other hand, $T^{t}\left(J_{m}\right) \bigcap B=T^{t}\left(J_{m, \delta}\right) \bigcap B$, where $J_{m, \delta}$ was defined in (23). It follows that for any $m \in \mathbb{N}$

$$
\chi_{V^{-}}\left(R_{\alpha}^{m}\left(x_{0}\right)\right) \lambda\left(J_{m, \delta}\right) \leq \lambda\left(J_{m} \cap T^{-t} B\right) \leq \chi_{V^{+}}\left(R_{\alpha}^{m}\left(x_{0}\right)\right) \lambda\left(J_{m, \delta}\right) .
$$

Since $J=\bigcup_{m \in \mathbb{N}} J_{m}$ this implies

$$
\sum_{m \in \mathbb{N}} \chi_{V^{-}}\left(R_{\alpha}^{m}\left(x_{0}\right)\right) \lambda\left(J_{m, \delta}\right) \leq \lambda\left(J \cap T^{-t} B\right) \leq \sum_{m \in \mathbb{N}} \chi_{V^{+}}\left(R_{\alpha}^{m}\left(x_{0}\right)\right) \lambda\left(J_{m, \delta}\right) .
$$

From the third point in Lemma 3.2 the foregoing becomes

$$
\sum_{m=m_{1}+1}^{m_{2}-1} \chi_{V^{-}}\left(R_{\alpha}^{m}\left(x_{0}\right)\right) \lambda\left(J_{m, \delta}\right) \leq \lambda\left(J \cap T^{-t} B\right) \leq \sum_{m=m_{1}}^{m=m_{2}} \chi_{V^{+}}\left(R_{\alpha}^{m}\left(x_{0}\right)\right) \lambda\left(J_{m, \delta}\right) .
$$

We use Lemma 3.2 again to obtain

$$
\begin{aligned}
\left(1-q_{n+1}^{-\eta}\right) \frac{\delta|J|}{\Delta \varphi_{m_{1} \mid J}} & \sum_{m=m_{1}+1}^{m_{2}-1} \chi_{V^{-}}\left(R_{\alpha}^{m}\left(x_{0}\right)\right) \\
& \leq \lambda\left(J \cap T^{-t} B\right) \leq\left(1+q_{n+1}^{-\eta}\right) \frac{\delta|J|}{\Delta \varphi_{m_{1} \mid J}} \sum_{m=m_{1}}^{m=m_{2}} \chi_{V^{+}}\left(R_{\alpha}^{m}\left(x_{0}\right)\right) .
\end{aligned}
$$

In the proof of Lemma 3.3, we obtained

$$
m_{2}-m_{1} \geq q_{n+1}^{\gamma-15 \eta} .
$$

TOME $129-2001-\mathrm{N}^{\mathrm{O}} 4$ 
Since $\eta \ll \gamma$ as well as $\epsilon \ll \gamma$, this implies on the one hand that

$$
\left(m_{2}-m_{1}\right)^{2 \epsilon} \leq q_{n+1}^{-2 \eta}\left(m_{2}-m_{1}\right)
$$

and on the other hand that if $n$ is large enough $m_{2}-m_{1}$ is large enough so that Lemma 3.4 applies to $V^{+}$and $V^{-}$; in conclusion we have

$$
\left|\sum_{i=0}^{m_{2}-m_{1}} \chi_{V^{+}}\left(R_{\alpha}^{i}\left(x_{0}\right)\right)-\left(m_{2}-m_{1}\right)\right| V^{+}|| \leq q_{n+1}^{-2 \eta}\left(m_{2}-m_{1}\right)\left|V^{+}\right|
$$

with a similar equation for $V^{-}$. Coming back to the estimation of $\lambda\left(J \cap T^{-t} B\right)$, we now have

$$
\begin{aligned}
& \left(1-q_{n+1}^{-\eta}\right) \frac{\delta|J|}{\Delta \varphi_{m_{1} \mid J}}\left(m_{2}-m_{1}\right)\left(1-q_{n+1}^{-2 \eta}\right)\left|V^{-}\right| \\
& \quad \leq \lambda\left(J \cap T^{-t} B\right) \leq\left(1+q_{n+1}^{-\eta}\right) \frac{\delta|J|}{\Delta \varphi_{m_{1} \mid J}}\left(m_{2}-m_{1}\right)\left(1+q_{n+1}^{-2 \eta}\right)\left|V^{+}\right| .
\end{aligned}
$$

Using Lemma 3.3 we obtain

$$
\begin{aligned}
& \left(1-q_{n+1}^{-\eta}\right)\left(1-q_{n+1}^{-\eta}\right)\left(1-2 q_{n+1}^{-\eta}\right) \delta|J| \cdot\left|V^{-}\right| \\
& \quad \leq \lambda\left(J \cap T^{-t} B\right) \leq\left(1+q_{n+1}^{-\eta}\right)\left(1+q_{n+1}^{-\eta}\right)\left(1+2 q_{n+1}^{-\eta}\right) \delta|J| \cdot\left|V^{+}\right| .
\end{aligned}
$$

Finally, with (33) and (34), the proof is over.

Acknowledgments. - I would like to thank François Ledrappier for useful comments and remarks. I also wish to thank the referee for his critical review of the manuscript.

\section{BIBLIOGRAPHY}

[1] FAYAD (B.R.) - Analytic mixing reparametrizations for irrational flows on the torus $\mathbb{T}^{n}, n \geq 3$, to appear in Ergodic Theory and Dynamical Flows.

[2] — Weak mixing for reparametrized linear flows on the torus, to appear in Ergodic Theory and Dynamical Flows.

[3] Khanin (K.M.) \& Sinai (YA.G.) - Mixing of some classes of special flows over rotations of the circle, Funkts. Anal. Prilozhen., t. 26 (1992), pp. 155169.

[4] KoČERGin (A.V.) - Mixing in special flows over a rearrangment of segments and in smooth flows on surfaces, Mat. USSR Sbornik, t. 25 (1975), pp. $471-502$.

[5] _ Nonsingular saddle points and absence of mixing, Math. Zametki, t. 19 (1976), pp. 453-468.

[6] Shklover (M.D.) - On dynamical systems on the torus with continuous spectrum, Izv. Vuzov, t. 10 (1967), pp. 113-124. 